\title{
Multiplicity results for $p$-Laplacian boundary value problem with jumping nonlinearities
}

\author{
Tacksun Jung $^{1 *}$ (D) and Q-Heung Choi ${ }^{2}$
}

${ }^{\text {*Correspondence: }}$

tsjung@kunsan.ac.kr

'Department of Mathematics,

Kunsan National University, Kunsan,

Korea

Full list of author information is

available at the end of the article

\begin{abstract}
We investigate the multiplicity of solutions for one-dimensional $p$-Laplacian Dirichlet boundary value problem with jumping nonlinearities. We obtain three theorems: The first states that there exists exactly one solution when nonlinearities cross no eigenvalue. The second guarantees that there exist exactly two solutions, exactly one solution and no solution, depending on the source term, when nonlinearities cross just the first eigenvalue. The third claims that there exist at least three solutions, exactly one solution and no solution, depending on the source term, when nonlinearities cross the first and second eigenvalues. We obtain the first and second theorem by considering the eigenvalues and the corresponding normalized eigenfunctions of the $p$-Laplacian eigenvalue problem, and the contraction mapping principle in the $p$-Lebesgue space (when $p \geq 2$ ). We obtain the third result by Leray-Schauder degree theory.
\end{abstract}

MSC: $35 \mathrm{~A} 01 ; 35 \mathrm{~A} 16 ; 35 J 30 ; 35 J 40 ; 35 J 60$

Keywords: $p$-Laplacian problem; $p$-Laplacian eigenvalue problem; Jumping nonlinearity; Contraction mapping principle; Leray-Schauder degree theory

\section{Introduction}

The $p$-Laplacian boundary value problems with $p$-growth conditions arise in applications of nonlinear elasticity theory, electro-rheological fluids, and in non-Newtonian fluid theory of a porous medium (cf. $[6,7,13])$. A typical model of elliptic equation with $p$-growth conditions is

$$
-\operatorname{div}\left((\alpha+|\nabla u|)^{p-2} \nabla u\right)=f(x, u) .
$$

In particular, when $\alpha=0$, the operator

$$
-\Delta_{p}=-\operatorname{div}\left((|\nabla u|)^{p-2} \nabla u\right)
$$

is called the $p$-Laplacian. In general, when $p=p(x)$, the $p(x)$-Laplacian problems are inhomogeneous, so they may have singular phenomena like $\inf \Lambda=0$, where $\Lambda$ is the set of the eigenvalues of the $p(x)$-Laplacian eigenvalue problem

$$
\begin{aligned}
& -\operatorname{div}\left((\alpha+|\nabla u|)^{p(x)-2} \nabla u\right)=\lambda|u|^{p(x)-2} u \text { in } \Omega, \\
& u=0 \text { on } \partial \Omega,
\end{aligned}
$$

(c) The Author(s) 2019. This article is distributed under the terms of the Creative Commons Attribution 4.0 International License (http://creativecommons.org/licenses/by/4.0/), which permits unrestricted use, distribution, and reproduction in any medium, provided you give appropriate credit to the original author(s) and the source, provide a link to the Creative Commons license, and indicate if changes were made. 
where $\Omega$ is a bounded domain in $R^{N}, N \geq 1$, with a smooth boundary $\partial \Omega$. But the eigenvalue problem when $\alpha=0, p(x)=p$ constant and $1<p<\infty$ has no singular phenomena, i.e., inf $\Lambda>0$. It was proved in [9] that the eigenvalue problem when $\alpha=0, p(x)=p$ constant and $1<p<\infty$ has a nondecreasing sequence of nonnegative eigenvalues $\lambda_{j}$, obtained by the Ljusternik-Schnirelman principle, tending to $\infty$ as $j \rightarrow \infty$, and the corresponding orthonomalized eigenfunctions $\phi_{j}, j=1,2, \ldots$, where the first eigenvalue $\lambda_{1}$ is positive and simple and only eigenfunctions associated with $\lambda_{1}$ do not change sign, the set of eigenvalues is closed, the first eigenvalue $\lambda_{1}$ is isolated. Thus there are two sequences of eigenfunctions $\left(\phi_{j}\right)_{j}$ and $\left(\mu_{j}\right)_{j}$ corresponding to the eigenvalues $\lambda_{j}$ such that the first eigenfunction $\phi_{1}>0$ in the sequence $\left(\phi_{j}\right)_{j}$ and the first eigenfunction $\mu_{1}<0$ in the sequence $\left(\mu_{j}\right)_{j}$.

Now in this paper, let $\phi_{1}$ be the first positive orthonormalized eigenfunction corresponding to $\lambda_{1}$.

In this paper we consider the multiplicity of solutions $u \in W^{1, p}(\Omega)$ for the following $p$ Laplacian Dirichlet boundary value problem with jumping nonlinearities when $N=1$ and $p(x)=p$

$$
\begin{aligned}
& -\left(\left|u^{\prime}\right|^{p-2} u^{\prime}\right)^{\prime}=b|u|^{p-2} u^{+}-a|u|^{p-2} u^{-}+s \phi_{1}^{p-1} \quad \text { in } \Omega, \\
& u=0 \quad \text { on } \partial \Omega,
\end{aligned}
$$

where $\Omega=(c, d) \subset R, c<d$, is an open interval, $2 \leq p<\infty$ and $p^{\prime}$ is given by $\frac{1}{p}+\frac{1}{p^{\prime}}=1$, $u^{+}=\max \{u, 0\}, u^{-}=-\min \{u, 0\}, s \in R, L^{p}(\Omega)$ is the $p$-Lebesgue space, with its dual space $L^{p^{\prime}}(\Omega)$, and $W^{1, p}(\Omega)$ is the Lebesgue-Sobolev space.

Our problems are characterized as a jumping problem, which was first suggested in the suspension bridge equation as a model of the nonlinear oscillations in a differential equation

$$
\begin{aligned}
& u_{t t}+K_{1} u_{x x x x}+K_{2} u^{+}=W(x)+\epsilon f(x, t), \\
& u(0, t)=u(L, t)=0, \quad u_{x x}(0, t)=u_{x x}(L, t)=0 .
\end{aligned}
$$

This equation represents a bending beam supported by cables under a load $f$. The constant $b$ represents the restoring force if the cables stretch. The nonlinearity $u^{+}$models the fact that cables resist expansion but do not resist compression. Choi and Jung (cf. [2-4]) and McKenna and Walter (cf. [12]) investigated the existence and multiplicity of solutions for the single nonlinear suspension bridge equation with a Dirichlet boundary condition. In [1], the authors investigated the multiplicity of solutions of a semilinear equation

$$
\begin{aligned}
& A u+b u^{+}-a u^{-}=f(x) \text { in } \Omega, \\
& u=0 \text { on } \Omega,
\end{aligned}
$$

where $\Omega$ is a bounded domain in $R^{n}, n \geq 1$, with a smooth boundary $\partial \Omega$, and $A$ is a secondorder linear partial differential operator when the forcing term is a multiple $s \phi_{1}, s \in R$, of the positive eigenfunction and the nonlinearity crosses eigenvalues.

Our main theorems are as follows: 


\section{Theorem 1.1}

(i) If $2 \leq p<\infty, a<b,-\infty<a, b<\lambda_{1}$, then (1.1) has exactly one nontrivial solution for all $s$ in a bounded interval. In particular, we have that if $2 \leq p<\infty, a<b$,

$-\infty<a, b<\lambda_{1}$ and $s>0$, then $u=\left(\frac{s}{\lambda_{1}-b}\right)^{\frac{1}{p-1}} \phi_{1}>0$ is a solution and if $-\infty<a, b<\lambda_{1}$ and $s<0$, then $u=\left(\frac{s}{\lambda_{1}-a}\right)^{\frac{1}{p-1}} \phi_{1}<0$ is a solution.

(ii) If $2 \leq p<\infty, a<b, \lambda_{j}<a, b<\lambda_{j+1}, s \in R$ is bounded, $j=1,2, \ldots$, (1.1) has exactly one nontrivial solution for all $s$ in a bounded interval.

\section{Theorem 1.2}

(i) If $1<p<\infty, a<b,-\infty<a<\lambda_{1}<b<\lambda_{2}, s \in R$ and $s>0$, then (1.1) has no solution,

(ii) If $1<p<\infty, a<b,-\infty<a<\lambda_{1}<b<\lambda_{2}, s \in R$ and $s=0$, then (1.1) has exactly one solution $u=0$.

(iii) If $2 \leq p<\infty, a<b,-\infty<a<\lambda_{1}<b<\lambda_{2}, s \in R$, then there exists $s_{1}<0$ such that for any $s$ with $s_{1} \leq s<0$, (1.1) has exactly two solutions.

\section{Theorem 1.3}

(i) If $1<p<\infty, a<b,-\infty<a<\lambda_{1}, \lambda_{2}<b<\lambda_{3}, s \in R$ and $s>0$, then (1.1) has no solution,

(ii) If $1<p<\infty, a<b,-\infty<a<\lambda_{1}, \lambda_{2}<b<\lambda_{3}, s \in R$ and $s=0$, then (1.1) has exactly one solution $u=0$.

(iii) If $2 \leq p<\infty, a<b,-\infty<a<\lambda_{1}, \lambda_{2}<b<\lambda_{3}, s \in R$, then there exists $s_{1}<0$ such that for any $s$ with $s_{1} \leq s<0$, (1.1) has at least three solutions.

For the proofs of (i) and (ii) of Theorem 1.1, (iii) of Theorem 1.2 and (iii) of Theorem 1.3, we use the contraction mapping principle under the condition $p \geq 2$ and direct computations. The outline of the proofs of Theorems 1.1, 1.2 and 1.3 is as follows: In Sect. 2, we introduce some preliminaries and prove Theorem 1.1 by direct computations using the first eigenfunction and the contraction mapping principle. In Sect. 3, we prove Theorem 1.2 by a similar method to that of Theorem 1.1. In Sect. 4, we prove Theorem 1.3 by direct computations using the first eigenfunction, contraction mapping principle and Leray-Schauder degree theory under the condition $p \geq 2$.

\section{Preliminaries and proof of Theorem 1.1}

Let $L^{p}(\Omega)$ be the Lebesgue space defined by

$$
L^{p}(\Omega)=\left\{u \mid u: \Omega \rightarrow R \text { is measurable, } \int_{c}^{d}|u|^{p} d x<\infty\right\}
$$

and $W^{1, p}(\Omega)$ be the Lebesgue-Sobolev space defined by

$$
W^{1, p}(\Omega)=\left\{u \in L^{p}(\Omega) \mid u^{\prime}(x) \in L^{p}(\Omega)\right\} .
$$

We introduce norms on $L^{p}(\Omega)$ and $W^{1, p}(\Omega)$, respectively, by

$$
\begin{aligned}
& \|u\|_{L^{p}(\Omega)}=\inf \left\{\lambda>\left.0\left|\int_{c}^{d}\right| \frac{u(x)}{\lambda}\right|^{p} \leq 1\right\} \\
& \|u\|_{W^{1, p}(\Omega)}=\left[\int_{c}^{d}\left|u^{\prime}(x)\right|^{p} d x\right]^{\frac{1}{p}}+\left[\int_{c}^{d}|u(x)|^{p} d x\right]^{\frac{1}{p}} .
\end{aligned}
$$


Let us define the operator $-L_{p}$ by

$$
-L_{p} u=-\left(\left|u^{\prime}\right|^{p-2} u^{\prime}\right)^{\prime}
$$

We first consider the problem:

$$
\begin{aligned}
& -\left(\left|u^{\prime}\right|^{p-2} u^{\prime}\right)^{\prime}=f(x) \text { in } \Omega, \\
& u=0 \quad \partial \Omega,
\end{aligned}
$$

where $1<p<\infty$ and $f \in L^{r}(\Omega), r>1$. Then (2.1) has a unique solution $u \in C^{1}(\bar{\Omega})$ which is of the form

$$
u(x)=\int_{c}^{x} g_{p}^{-1}\left(c_{f}-\int_{a}^{y} f(\tau) d \tau\right) d y,
$$

where $g_{p}(t)=|t|^{p-2} t$ for $t \neq 0, g_{p}(0)=0$ and its inverse $g_{p}^{-1}$ is $g_{p}^{-1}(t)=t^{\frac{1}{p-1}}$ if $t>0$ and $g_{p}^{-1}(t)=$ $-|t|^{\frac{1}{p-1}}$ if $t<0$, and $c_{f}$ is the unique constant such that $u(d)=0$. By [10, Lemma 2.1] or [11, Lemma 4.2], the solution operator $S$ is such that $S: L^{p}(\Omega) \rightarrow C^{1}(\bar{\Omega})$ is continuous and, by [5, Corollary 2.3], the embedding $S: L^{p}(\Omega) \rightarrow C(\bar{\Omega})$ is continuous and compact. By [8], we also have a Poincaré-type inequality.

Lemma 2.1 Let $1<p<\infty$. Then the embedding

$$
H^{1, p}(\Omega) \hookrightarrow L^{p}(\Omega)
$$

is continuous and compact, and for every $u \in C_{0}^{\infty}(\Omega)$ we have

$$
\|u\|_{L^{p}(\bar{\Omega})} \leq C\|u\|_{W^{1, p}(\bar{\Omega})},
$$

for a positive constant $C$ independent of $u$.

By Lemma 2.1, we obtain the following:

Lemma 2.2 Assume that $1<p<\infty, f(x, u) \in L^{p}(\Omega)$. Then the solutions of the problem

$$
\begin{aligned}
& -\left(\left|u^{\prime}\right|^{p-2} u^{\prime}\right)^{\prime}=f(x, u) \quad \text { in } L^{p}(\Omega), \\
& u=0 \quad \partial \Omega
\end{aligned}
$$

belong to $W^{1, p}(\Omega)$.

For given $v(u) \in L^{p}(\Omega)$ and $h(x) \in L^{p}(\Omega)$, the equation

$$
-L_{p} u=v(u)+h(x) \quad \text { in } L^{p}(\Omega)
$$

is equivalent to the equation

$$
u=\left(-L_{p}\right)^{-1}(v(u)+h(x)) .
$$


We observe that

$$
\begin{aligned}
\left\|\left(-L_{p}\right)^{-1}(v(u)+h(x))\right\|_{W^{1, p}(\Omega)} & \leq \frac{1}{\lambda_{1}}\left\|g_{p}^{-1}(v(u)+h(x))\right\|_{W^{1, p}(\Omega)} \\
& \leq \frac{1}{\lambda_{1}}\left\|(v(u)+h(x))^{\frac{1}{p-1}}\right\|_{L^{p}(\Omega)} .
\end{aligned}
$$

Proof of Theorem 1.1 (i) We assume that $-\infty<a, b<\lambda_{1}$. Let us choose $\mu>0$ and $\epsilon>0$ so that $-\mu+\epsilon<a, b<\lambda_{1}-\epsilon$, and choose $\tau=\frac{a+b}{2}$. Then problem (1.1) can be rewritten as

$$
-L_{p}-\tau|u|^{p-2} u=(b-\tau)|u|^{p-2} u^{+}-(a-\tau)|u|^{p-2} u^{-}+s \phi_{1}^{p-1},
$$

or equivalently,

$$
u=\left(-L_{p}-\tau g_{p}\right)^{-1}\left((b-\tau)|u|^{p-2} u^{+}-(a-\tau)|u|^{p-2} u^{-}+s \phi_{1}^{p-1}\right) .
$$

We have that

$$
\begin{aligned}
& \left\|\left(-L_{p}-\tau g_{p}\right)^{-1}\left((b-\tau)|u|^{p-2} u^{+}-(a-\tau)|u|^{p-2} u^{-}+s \phi_{1}^{p-1}\right)\right\|_{L^{p}(\Omega)} \\
& \quad \leq\left\|\frac{1}{\lambda_{1}-\tau} g_{p}^{-1}\left((b-\tau)|u|^{p-2} u^{+}-(a-\tau)|u|^{p-2} u^{-}+s \phi_{1}^{p-1}\right)\right\|_{L^{p}(\Omega)} .
\end{aligned}
$$

Let us set the right-hand side of (2.3) as

$$
T(u)=\left(-L_{p}-\tau g_{p}\right)^{-1}\left((b-\tau)|u|^{p-2} u^{+}-(a-\tau)|u|^{p-2} u^{-}+s \phi_{1}^{p-1}\right) .
$$

Then $T(u)$ satisfies

$$
\begin{aligned}
&\|T(u)-T(v)\|_{L^{p}(\Omega)} \\
&=\|\left(-L_{p}-\tau g_{p}\right)^{-1}\left((b-\tau)|u|^{p-2} u^{+}-(a-\tau)|u|^{p-2} u^{-}+s \phi_{1}^{p-1}\right) \\
&-\left(-L_{p}-\tau g_{p}\right)^{-1}\left((b-\tau)|v|^{p-2} v^{+}-(a-\tau)|v|^{p-2} v^{-}+s \phi_{1}^{p-1}\right) \|_{L^{p}(\Omega)} \\
& \leq \frac{1}{\lambda_{1}-\tau} \| g_{p}^{-1}\left((b-\tau)|u|^{p-2} u^{+}-(a-\tau)|u|^{p-2} u^{-}+s \phi_{1}^{p-1}\right) \\
&-g_{p}^{-1}\left((b-\tau)|v|^{p-2} \nu^{+}-(a-\tau)|v|^{p-2} v^{-}+s \phi_{1}^{p-1}\right) \|_{L^{p}(\Omega)} \\
&= \frac{1}{\lambda_{1}-\tau}\left\|g_{p}^{-1}\left(\frac{b-a}{2}|u|^{p-1}+s \phi_{1}^{p-1}\right)-g_{p}^{-1}\left(\frac{b-a}{2}|v|^{p-1}+s \phi_{1}^{p-1}\right)\right\|_{L^{p}(\Omega)} .
\end{aligned}
$$

Since $|\cdot|^{p-1}$ is continuous, for given $u \in W^{1, p}(\Omega)$, there exists $v \in W^{1, p}(\Omega)$ in a small neighborhood of $u$ such that $\frac{b-a}{2}|u|^{p-1}+s \phi_{1}^{p-1}$ and $\frac{b-a}{2}|v|^{p-1}+s \phi_{1}^{p-1}$ have the same sign. We can also check that for any $2 \leq p<\infty, s<0$ and for any $u$ and $v$, where $v$ is in a small neighborhood of $u$ such that $\frac{b-a}{2}|u|^{p-1}+s \phi_{1}^{p-1}$ and $\frac{b-a}{2}|v|^{p-1}+s \phi_{1}^{p-1}$ have the same sign, we have

$$
\begin{aligned}
& \left\|g_{p}^{-1}\left(\frac{b-a}{2}|u|^{p-1}+s \phi_{1}^{p-1}\right)-g_{p}^{-1}\left(\frac{b-a}{2}|v|^{p-1}+s \phi_{1}^{p-1}\right)\right\|_{L^{p}(\Omega)} \\
& \quad \leq\left\|g_{p}^{-1}\left(\frac{b-a}{2}|u|^{p-1}\right)-g_{p}^{-1}\left(\frac{b-a}{2}|v|^{p-1}\right)\right\|_{L^{p}(\Omega)} .
\end{aligned}
$$


Thus for any $u$ and $v$, where $v$ is in a small neighborhood of $u$ such that $\frac{b-a}{2}|u|^{p-1}+s \phi_{1}^{p-1}$ and $\frac{b-a}{2}|\nu|^{p-1}+s \phi_{1}^{p-1}$ have the same sign, we have

$$
\begin{aligned}
& \|T(u)-T(v)\|_{L^{p}(\Omega)} \\
& \quad \leq \frac{1}{\lambda_{1}-\tau}\left\|g_{p}^{-1}\left(\frac{b-a}{2}|u|^{p-1}+s \phi_{1}^{p-1}\right)-g_{p}^{-1}\left(\frac{b-a}{2}|v|^{p-1}+s \phi_{1}^{p-1}\right)\right\|_{L^{p}(\Omega)} \\
& \quad \leq \frac{1}{\lambda_{1}-\tau}\left(\frac{b-a}{2}\right)^{\frac{1}{p-1}}\left\|g_{p}^{-1}\left(\left.u\right|^{p-1}\right)-g_{p}^{-1}\left(|v|^{p-1}\right)\right\|_{L^{p}(\Omega)} \\
& \quad=\frac{\left(\frac{b-a}{2}\right)^{\frac{1}{p-1}}}{\lambda_{1}-\tau}\||u|-|v|\|_{L^{p}(\Omega)} \leq \frac{\left(\frac{b-a}{2}\right)^{p-1}}{\lambda_{1}-\tau}\|u-v\|_{L^{p}(\Omega)} .
\end{aligned}
$$

Since $\lambda_{1}-\tau>\frac{b-a}{2}$ and $2 \leq p<\infty$, we have $\frac{\left(\frac{b-a}{2}\right)^{\frac{1}{p-1}}}{\lambda_{1}-\tau}<1$. Thus $T$ is a contraction mapping on $L^{p}(\Omega)$. Thus $T$ has a unique solution in $L^{p}(\Omega)$. Thus (1.1) has a unique solution.

(ii) We assume that $\lambda_{j}<a, b<\lambda_{j+1}, j=1,2, \ldots$ Let us choose $\epsilon>0$ so that $\lambda_{j}+\epsilon<a, b<$ $\lambda_{j+1}-\epsilon$. Let us set $\tau=\frac{a+b}{2}$. Then (1.1) can be rewritten as

$$
u=\left(-L_{p}-\tau g_{p}\right)^{-1}\left((b-\tau)|u|^{p-2} u^{+}-(a-\tau)|u|^{p-2} u^{-}+s \phi_{1}^{p-1}\right) .
$$

We observe that

$$
\begin{aligned}
& \left\|\left(-L_{p}-\tau g_{p}\right)^{-1}\left((b-\tau)|u|^{p-2} u^{+}-(a-\tau)|u|^{p-2} u^{-}\right)\right\|_{L^{P}(\Omega)} \\
& \quad \leq\left\|\left(\frac{2}{\lambda_{j+1}-\lambda_{j}}\right)^{-1} g_{p}^{-1}\left((b-\tau)|u|^{p-2} u^{+}-(a-\tau)|u|^{p-2} u^{-}\right)\right\|_{L^{P}(\Omega)} .
\end{aligned}
$$

By the same process as that of the proof of Theorem 1.1(i), the mapping on $L^{p}(\Omega)$ given by

$$
N(u)=\left(-L_{p}-\tau g_{p}\right)^{-1}\left((b-\tau)|u|^{p-2} u^{+}-(a-\tau)|u|^{p-2} u^{-}+s \phi_{1}^{p-1}\right)
$$

satisfies

$$
\begin{array}{rl}
\| N & N(u)-N(v) \|_{L^{p}(\Omega)} \\
= & \|\left(-L_{p}-\tau g_{p}\right)^{-1}\left(( b - \tau ) \left(|u|^{p-2} u^{+}-(a-\tau)\left(|u|^{p-2} u^{-}+s \phi_{1}^{p-1}\right)\right.\right. \\
& -\left(-L_{p}-\tau g_{p}\right)^{-1}\left(( b - \tau ) \left(|v|^{p-2} v^{+}-(a-\tau)\left(|v|^{p-2} v^{-}+s \phi_{1}^{p-1}\right) \|_{L^{p}(\Omega)}\right.\right. \\
= & \frac{2}{\lambda_{j+1}-\lambda_{j}} \| g_{p}^{-1}\left(\frac{b-a}{2}\left(|u|^{p-1}+s \phi_{1}^{p-1}\right)-g_{p}^{-1}\left(\frac{b-a}{2}\left(|v|^{p-1}+s \phi_{1}^{p-1}\right) \|_{L^{p}(\Omega)}\right.\right. \\
\leq & \frac{2}{\lambda_{j+1}-\lambda_{j}} \| g_{p}^{-1}\left(\frac{b-a}{2}\left(|u|^{p-1}\right)-g_{p}^{-1}\left(\frac{b-a}{2}\left(|v|^{p-1}\right) \|_{L^{p}(\Omega)}\right.\right. \\
\leq & \frac{2}{\lambda_{j+1}-\lambda_{j}}\left(\frac{b-a}{2}\right)^{\frac{1}{p-1}}\||u|-|v|\|_{L^{p}(\Omega)} \leq \frac{2}{\lambda_{j+1}-\lambda_{j}}\left(\frac{b-a}{2}\right){ }^{\frac{1}{p-1}}\|u-v\|_{L^{p}(\Omega)}
\end{array}
$$

for any $u$ and $v$, where $v$ is in a small neighborhood of $u$ such that $\frac{b-a}{2}|u|^{p-1}+s \phi_{1}^{p-1}$ and $\frac{b-a}{2}|\nu|^{p-1}+s \phi_{1}^{p-1}$ have the same sign, and $2 \leq p<\infty$. Since $\frac{\lambda_{j+1}-\lambda_{j}}{2}>\frac{b-a}{2}$ and $2 \leq p<\infty$, we 
have $\frac{2}{\lambda_{j+1}-\lambda_{j}}\left(\frac{b-a}{2}\right)^{\frac{1}{p-1}}<1$. It follows that $N$ is a contraction mapping on $L^{p}(\Omega)$. Therefore $N$ has a unique solution in $L^{p}(\Omega)$. Thus (1.1) has a unique solution.

\section{Proof of Theorem $\mathbf{1 . 2}$}

(i) (For the case $s>0$ ) We assume that $1<p<\infty, a<b,-\infty<a<\lambda_{1}<b<\lambda_{2}$ and $s>0$. Then (3.1) can be rewritten as

$$
-\left(\left|u^{\prime}\right|^{p-2} u^{\prime}\right)^{\prime}-\lambda_{1}|u|^{p-2} u=\left(b-\lambda_{1}\right)|u|^{p-2} u^{+}-\left(a-\lambda_{1}\right)|u|^{p-2} u^{-}+s \phi_{1}^{p-1} .
$$

Taking the inner product with $\phi_{1}$, we have

$$
\left\langle-\left(\left|u^{\prime}\right|^{p-2} u^{\prime}\right)^{\prime}-\lambda_{1}|u|^{p-2} u, \phi_{1}\right\rangle=\left\langle\left(b-\lambda_{1}\right)|u|^{p-2} u^{+}-\left(a-\lambda_{1}\right)|u|^{p-2} u^{-}+s \phi_{1}^{p-1}, \phi_{1}\right\rangle
$$

The left-hand side of (3.1) is equal to 0 . On the other hand, the right-hand side of (3.1) is positive because $b-\lambda_{1}>0,-\left(a-\lambda_{1}\right)>0$ and $s \phi_{1}^{p-1}>0$ for $s>0$. Thus if $s>0$, then there is no solution for (1.1).

(ii) (For the case $s=0$ ) If $s=0$, then (3.2) is reduced to the equation

$$
\left\langle-\left(\left|u^{\prime}\right|^{p-2} u^{\prime}\right)^{\prime}-\lambda_{1}|u|^{p-2} u, \phi_{1}\right\rangle=\left\langle\left(b-\lambda_{1}\right)|u|^{p-2} u^{+}-\left(a-\lambda_{1}\right)|u|^{p-2} u^{-}, \phi_{1}\right\rangle
$$

i.e.,

$$
\begin{aligned}
& \int_{c}^{d}\left[\left(-\left(\left|u^{\prime}\right|^{p-2} u^{\prime}\right)^{\prime}-\lambda_{1}|u|^{p-2} u\right) \phi_{1}\right] d x \\
& \quad=0=\int_{c}^{d}\left[\left(\left(b-\lambda_{1}\right)|u|^{p-2} u^{+}-\left(a-\lambda_{1}\right)|u|^{p-2} u^{-}\right) \phi_{1}\right] d x .
\end{aligned}
$$

Since $b-\lambda_{1}>0$ and $-\left(a-\lambda_{1}\right)>0$, the only possibility for (3.3) to hold is that $u=0$.

(iii) (For the case $s<0$ ) We assume that $2 \leq p<\infty, a<b,-\infty<a<\lambda_{1}<b<\lambda_{2}$ and $s<0$. Let $V$ be a subspace of $L^{p}(\Omega)$ spanned by $\phi_{1}$ and $W$ be the orthogonal complement of $V$ in $L^{p}(\Omega)$. Then

$$
L^{p}(\Omega)=V \oplus W .
$$

Let $P$ be a orthogonal projection in $L^{p}(\Omega)$ onto $V$ and $I-P$ be the orthogonal projection onto $W$. Then

$$
P u=\left(\int_{c}^{d} u \phi_{1}\right) \phi_{1} \quad \text { for all } u \in L^{P}(\Omega) .
$$

Let $u \in L^{p}(\Omega)$. Then $u$ can be written as

$$
u=v+w, \quad v=P u, \quad w=(I-P) w .
$$

We note that $P$ commutes with $D=\frac{d}{d x}$. Thus (1.1) is equivalent to a pair of equations

$$
\begin{aligned}
(I-P)\left(-\left(\left|(v+w)^{\prime}\right|^{p-2}(v+w)^{\prime}\right)^{\prime}\right) \\
\quad=(I-P)\left(b|v+w|^{p-2}(v+w)^{+}-a|v+w|^{p-2}(v+w)^{-}\right),
\end{aligned}
$$




$$
\begin{aligned}
P( & \left.-\left(\left|(v+w)^{\prime}\right|^{p-2}(v+w)^{\prime}\right)^{\prime}\right) \\
\quad & =P\left(b|v+w|^{p-2}(v+w)^{+}-a|v+w|^{p-2}(v+w)^{-}+s \phi_{1}^{p-1}\right) .
\end{aligned}
$$

We claim that for fixed $v \in V$, (3.4) has a unique solution $w(v)$ when $2 \leq p \leq \infty$. In fact, we suppose that (3.4) has two solutions $w_{1}, w_{2}$ for fixed $v \in V$. Let us set $\alpha_{v}(w)=b \mid v+$ $\left.w\right|^{p-2}(v+w)^{+}-a|v+w|^{p-2}(v+w)^{-}$. Then we have

$$
\begin{gathered}
(I-P)\left[\left(-\left(\left|\left(v+w_{1}\right)^{\prime}\right|^{p-2}\left(v+w_{1}\right)^{\prime}\right)^{\prime}\right)-\left(-\left(\left|\left(v+w_{2}\right)^{\prime}\right|^{p-2}\left(v+w_{2}\right)^{\prime}\right)^{\prime}\right)\right] \\
=(I-P)\left[\left(b\left|v+w_{1}\right|^{p-2}\left(v+w_{1}\right)^{+}-a\left|v+w_{1}\right|^{p-2}\left(v+w_{1}\right)^{-}\right)\right. \\
\left.-\left(b\left|v+w_{2}\right|^{p-2}\left(v+w_{2}\right)^{+}-a\left|v+w_{2}\right|^{p-2}\left(v+w_{2}\right)^{-}\right)\right] .
\end{gathered}
$$

Taking the inner product of (3.6) with $w_{1}-w_{2}$, we have

$$
\begin{aligned}
\langle(I-P) & {\left.\left[\left(-\left(\left|\left(v+w_{1}\right)^{\prime}\right|^{p-2}\left(v+w_{1}\right)^{\prime}\right)^{\prime}\right)-\left(-\left(\left|\left(v+w_{2}\right)^{\prime}\right|^{p-2}\left(v+w_{2}\right)^{\prime}\right)^{\prime}\right)\right], w_{1}-w_{2}\right\rangle } \\
= & \left\langle( I - P ) \left[\left(b\left|v+w_{1}\right|^{p-2}\left(v+w_{1}\right)^{+}-a\left|v+w_{1}\right|^{p-2}\left(v+w_{1}\right)^{-}\right)\right.\right. \\
& \left.\left.-\left(b\left|v+w_{2}\right|^{p-2}\left(v+w_{2}\right)^{+}-a\left|v+w_{2}\right|^{p-2}\left(v+w_{2}\right)^{-}\right)\right], w_{1}-w_{2}\right\rangle .
\end{aligned}
$$

The right-hand side of (3.7) is equal to

$$
\begin{aligned}
\langle(I-P) & {\left[\left(b\left|v+w_{1}\right|^{p-2}\left(v+w_{1}\right)^{+}-a\left|v+w_{1}\right|^{p-2}\left(v+w_{1}\right)^{-}\right)\right.} \\
& \left.\left.-\left(b\left|v+w_{2}\right|^{p-2}\left(v+w_{2}\right)^{+}-a\left|v+w_{2}\right|^{p-2}\left(v+w_{2}\right)^{-}\right)\right], w_{1}-w_{2}\right\rangle \\
\leq & (p-1) b \int_{c}^{d}\left[[I-P]\left|v+w_{1}+\theta\left(w_{2}-w_{1}\right)\right|^{p-2}\left(w_{1}-w_{2}\right)^{2}\right] d x
\end{aligned}
$$

for $0<\theta<1$. On the other hand, the left-hand side of (3.6) is equal to

$$
\begin{aligned}
& \left\langle(I-P)\left[\left(-\left(\left|\left(v+w_{1}\right)^{\prime}\right|^{p-2}\left(v+w_{1}\right)^{\prime}\right)^{\prime}\right)-\left(-\left(\left|\left(v+w_{2}\right)^{\prime}\right|^{p-2}\left(v+w_{2}\right)^{\prime}\right)^{\prime}\right)\right], w_{1}-w_{2}\right\rangle \\
& \quad=(p-1) \int_{c}^{d}\left[(I-P)\left(\left|\left(v+w_{1}\right)^{\prime}+\theta\left(w_{2}-w_{1}\right)^{\prime}\right|^{p-2}\left(\left(w_{1}-w_{2}\right)^{\prime}\right)^{2}\right)\right] d x \\
& \quad \geq(p-1) \lambda_{2} \int_{c}^{d}\left[(I-P)\left(\left|\left(v+w_{1}\right)+\theta\left(w_{2}-w_{1}\right)\right|^{p-2}\left(w_{1}-w_{2}\right)^{2}\right)\right] d x
\end{aligned}
$$

by mean value theorem. On the other hand, by (3.8) and (3.9), we have

$$
\begin{aligned}
& (p-1) \lambda_{2} \int_{c}^{d}\left[(I-P)\left(\left|\left(v+w_{1}\right)+\theta\left(w_{2}-w_{1}\right)\right|^{p-2}\left(w_{1}-w_{2}\right)^{2}\right)\right] d x \\
& \leq(p-1) b \int_{c}^{d}\left[[I-P]\left|v+w_{1}+\theta\left(w_{2}-w_{1}\right)\right|^{p-2}\left(w_{1}-w_{2}\right)^{2}\right] d x
\end{aligned}
$$

which is a contradiction because $b<\lambda_{2}$. Thus $w_{1}=w_{2}$. Thus for fixed $v \in V$, every solution of (3.4) is unique. We note that $w=0$ is a solution of (3.4) for every $v \in V=P H, v>0$ or $v<0$ everywhere in $\Omega$. If $v>0$, then

$$
(I-P)\left(-\left(\left|(v+w)^{\prime}\right|^{p-2}(v+w)^{\prime}\right)^{\prime}\right)=(I-P)\left(b|v|^{p-2} v^{+}-a|v|^{p-2} v^{-}+s \phi_{1}^{p-1}\right) .
$$


If $v>0$, then $(I-P)\left(b|v|^{p-2} v^{+}-a|v|^{p-2} v^{-}+s \phi_{1}^{p-1}\right)=(I-P) b|v|^{p-2} v=0$. If $v<0$, then $(I-$ $P)\left(b|v|^{p-2} v^{+}-a|v|^{p-2} v^{-}+s \phi_{1}^{p-1}\right)=(I-P)\left(a|v|^{p-2} v+s \phi_{1}^{p-1}\right)=0$. Thus (1.1) is reduced to

$$
P\left(-\left(\left|v^{\prime}\right|^{p-2} v^{\prime}\right)^{\prime}\right)=P\left(b|v|^{p-2} v^{+}-a|v|^{p-2} v^{-}+s \phi_{1}^{p-1}\right),
$$

where $v=c \phi_{1}, c \in R$.

If $c>0$, then

$$
\lambda c^{p-1}=b c^{p-1}+s, \quad c^{p-1}=\frac{s}{\lambda_{1}-b}
$$

If $c<0$, then

$$
\lambda|c|^{p-2} c=a|c|^{p-2} c+s, \quad|c|^{p-2} c=\frac{s}{\lambda_{1}-a} .
$$

Thus (1.1) has exactly two solutions.

\section{Proof of Theorem 1.3}

Lemma 4.1 (A priori bound) Assume that $1<p<\infty,-\infty<a<\lambda_{1}, \lambda_{2}<b<\lambda_{3}, s \in R$. Then there exist $s_{1}<0, s_{2}>0$ and a constant $C>0$ depending only on $a$ and $b$ such that for any $s$ with $s_{1} \leq s \leq s_{2}$, any solution $u$ of $(1.1)$ satisfies $\|u\|_{W^{1, p}(\Omega)}<C$.

Proof Let $u$ be any solution of (1.1). Suppose that any solution of (1.1) is not bounded. Then there exists a sequence $\left(u_{n}\right)_{n}$ such that $\left\|u_{n}\right\|_{W^{1, p}(\Omega)} \rightarrow \infty$ so that

$$
-\left(\left|u_{n}^{\prime}\right|^{p-2} u_{n}^{\prime}\right)^{\prime}=b\left|u_{n}\right|^{p-2} u_{n}^{+}-a\left|u_{n}\right|^{p-2} u_{n}^{-}+s \phi_{1}^{p-1} \quad \text { in } \Omega
$$

or equivalently,

$$
u_{n}=\left(-L_{p}\right)^{-1}\left(b\left|u_{n}\right|^{p-2} u_{n}^{+}-a\left|u_{n}\right|^{p-2} u_{n}^{-}+s \phi_{1}^{p-1}\right) \quad \text { in } \Omega .
$$

Let us set $w_{n}=\frac{u_{n}}{\left\|u_{n}\right\|_{W^{1, p}(\Omega)}}$. Then $\left(w_{n}\right)_{n}$ is bounded, so by passing to a subsequence if necessary, which we still denote by $\left(w_{n}\right)_{n}$, we get that $\left(w_{n}\right)_{n} \rightarrow w$ weakly for some $w$ in $W^{1, p}(\Omega)$. Dividing (4.1) by $\left\|u_{n}\right\|_{W^{1, p}(\Omega)}^{p-1}$, we have

$$
\frac{-\left(\left|u_{n}^{\prime}\right|^{p-2} u_{n}^{\prime}\right)^{\prime}}{\left\|u_{n}\right\|_{W^{1, p}(\Omega)}^{p-1}}=b \frac{\left|u_{n}\right|^{p-2} u_{n}^{+}}{\left\|u_{n}\right\|_{W^{1, p}(\Omega)}^{p-1}}-a \frac{\left|u_{n}\right|^{p-2} u_{n}^{-}}{\left\|u_{n}\right\|_{W^{1, p}(\Omega)}^{p-1}}+\frac{s \phi_{1}^{p-1}}{\left\|u_{n}\right\|_{W^{1, p}(\Omega)}^{p-1}} \quad \text { in } \Omega
$$

i.e.,

$$
w_{n}=\left(-L_{p}\right)^{-1}\left(b\left|w_{n}\right|^{p-2} w_{n}^{+}-a\left|w_{n}\right|^{p-2} w_{n}^{-}+\frac{s \phi_{1}^{p-1}}{\left\|u_{n}\right\|_{W^{1, p}(\Omega)}^{p-1}}\right) \quad \text { in } \Omega .
$$

Since, by Lemma 2.1, the embedding $W^{1, p}(\Omega) \hookrightarrow L^{p}(\Omega)$ is compact, and $\left(-L_{p}\right)^{-1}$ is compact operator, $\left(w_{n}\right)_{n} \rightarrow w$ strongly in $W^{1, p}(\Omega)$. Taking the limit of (4.2) as $n \rightarrow \infty$, we have

$$
-\left(\left|w^{\prime}\right|^{p-2} w^{\prime}\right)^{\prime}=b|w|^{p-2} w^{+}-a|w|^{p-2} w^{-} .
$$


By Theorem 1.1(i), (4.3) has only the trivial solution, which is absurd, because $\|w\|_{W^{1, p}(\Omega)}=1$. Thus the lemma is proved.

We shall consider the Leray-Schauder degree on a large ball.

Lemma 4.2 Assume that $1<p<\infty,-\infty<a<\lambda_{1}, \lambda_{2}<b<\lambda_{3}$. Then there exist a constant $R>0$ depending on $a, b, s, s_{1}<0$ and $s_{2}>0$ such that for any $s$ with $s_{1} \leq s \leq s_{2}$, the LeraySchauder degree

$$
d_{\mathrm{LS}}\left(u-\left(-L_{p}\right)^{-1}\left(b|u|^{p-2} u^{+}-a|u|^{p-2} u^{-}+s \phi_{1}^{p-1}\right), B_{R}(0), 0\right)=0
$$

where $-L_{p} u=-\left(\left|u^{\prime}\right|^{p-2} u^{\prime}\right)^{\prime}$

Proof Let us consider the homotopy

$$
F(x, u)=u-\left(-L_{p}\right)^{-1}\left(b|u|^{p-2} u^{+}-a|u|^{p-2} u^{-}+s \phi_{1}^{p-1}\right) .
$$

By Theorem 1.3(ii), for any $s>0,(1.1)$ has no solution. Thus there exist $s_{2}>0$ and a large $R>0$ such that (4.3) has no zero in $B_{R}(0)$ for any $s \geq s_{2}$, and by the a priori bound in Lemma 4.1, there exists $s_{1}<0$ such that for any $s$ with $s_{1} \leq s \leq s_{2}$, all solutions of

$$
u-\left(-L_{p}\right)^{-1}\left(b|u|^{p-2} u^{+}-a|u|^{p-2} u^{-}+s \phi_{1}^{p-1}\right)=0
$$

satisfy $\|u\|_{W^{1, p}(\Omega)} \leq R$ and (4.3) has no zero on $\partial B_{R}$ for any $s$ with $s_{1} \leq s \leq s_{2}$. Since

$$
d_{\mathrm{LS}}\left(u-\left(-L_{p}\right)^{-1}\left(b|u|^{p-2} u^{+}-a|u|^{p-2} u^{-}+s_{2} \phi_{1}^{p-1}\right), B_{R}(0), 0\right)=0
$$

by homotopy arguments, for any any $s$ with $s_{1} \leq s \leq s_{2}$, we have

$$
\begin{aligned}
& d_{\mathrm{LS}}\left(u-\left(-L_{p}\right)^{-1}\left(b|u|^{p-2} u^{+}-a|u|^{p-2} u^{-}+s \phi_{1}^{p-1}\right), B_{R}(0), 0\right) \\
& \quad=d_{\mathrm{LS}}\left(u-\left(-L_{p}\right)^{-1}\left(b|u|^{p-2} u^{+}-a|u|^{p-2} u^{-}+s \phi_{1}^{p-1}+\lambda\left(s_{2}-s\right) \phi_{1}^{p-1}\right), B_{R}(0), 0\right) \\
& \quad=d_{\mathrm{LS}}\left(u-\left(-L_{p}\right)^{-1}\left(b|u|^{p-2} u^{+}-a|u|^{p-2} u^{-}+s_{2} \phi_{1}^{p-1}\right), B_{R}(0), 0\right)=0,
\end{aligned}
$$

for any $0 \leq \lambda \leq 1$. Thus the lemma is proved.

Lemma 4.3 Let $K$ be a compact set in $L^{p}(\Omega)$. Let $\xi>0$ a.e. Then there exists a modulus of continuity $\alpha: R \rightarrow R$ depending only on $K$ and $\xi$ such that

$$
\left\|\mid\left(|\tau|-\frac{\xi}{\eta}\right)^{+}\right\|_{L^{p}(\Omega)} \leq \alpha(\eta) \text { for all } \tau \in K \text {. }
$$

It follows that

$$
\left\|\mid(\eta \tau+\xi)^{-}\right\|_{L^{p}(\Omega)} \leq \eta \alpha(\eta)
$$

and

$$
\left\|\mid(\eta \tau-\xi)^{+}\right\|_{L^{p}(\Omega)} \leq \eta \alpha(\eta) \quad \text { for all } \tau \in K
$$


Proof For any $\tau \in K$, let $\tau_{n}=\left(|\tau|-\frac{\xi}{\eta}\right)^{+}$. Since $0 \leq \tau_{n} \leq|\tau|$ and $\tau_{n}(x) \rightarrow 0$ as $\eta \rightarrow 0$ a.e., it follows that $\left\|\tau_{n}\right\|_{L^{p}(\Omega)} \rightarrow 0$ for all $\tau \in K$. We claim that, for a given $\epsilon>0$, there exists $\delta>0$ such that if $\tau \in K$, then $\left\|\tau_{n}\right\|_{L^{p}(\Omega)} \leq 2 \epsilon$ for all $\eta \in[0, \delta]$. Choose $\left\{\tau_{i}, i=1, \ldots, N\right\}$ as an $\epsilon$ net for $K$. Choose $\delta$ so that $\left\|\left(\tau_{i}\right)_{\delta}\right\|_{L^{p}(\Omega)}<\epsilon$ for $i=1, \ldots, N$. Then for any $\tau \in K$, there exists $\tau_{k}$, $\alpha,\|\alpha\|_{L^{P}(\Omega)}<\epsilon$ that $\tau=\tau_{K}+\alpha$. Since $(u+v)^{+} \leq u^{+}+v^{+}$, we have $\left\|\tau_{\delta}\right\|_{L^{P}(\Omega)} \leq\left(\tau_{K}\right)_{\delta}+|\alpha|$ and therefore $\left\|\tau_{\eta}\right\|_{L^{P}(\Omega)} \leq\left\|\tau_{\delta}\right\|_{L^{P}(\Omega)}+\|\alpha\|_{L^{p}(\Omega)} \leq 2 \epsilon$.

Lemma 4.4 Assume that $1<p<\infty,-\infty<a<\lambda_{1}, \lambda_{2}<b<\lambda_{3}$. Then there exist a constant $R>0$ depending on $a, b$, s and $s_{1}<0$ such that for $s_{1} \leq s<0$, the Leray-Schauder degree

$$
d_{\mathrm{LS}}\left(u-(-L)^{-1}\left(b|u|^{p-2} u^{+}-a|u|^{p-2} u^{-}+s \phi_{1}^{p-1}\right), B_{R_{0}}\left(u_{0}\right), 0\right)=1,
$$

where $u_{0}=\left(\frac{s}{\lambda_{1}-b}\right)^{\frac{1}{p-1}} \phi_{1}>0$ is a positive solution of $(1.1)$.

Proof Let us set $M=\left(-L_{p}-b g_{p}\right)^{-1}$. Then (1.1) can be rewritten as

$$
\left(-L_{p}-b g_{p}\right)(u)=b|u|^{p-2} u^{+}-a|u|^{p-2} u^{-}-b|u|^{p-2} u+s \phi_{1}^{p-1},
$$

or equivalently,

$$
u=M\left(b|u|^{p-2} u^{+}-a|u|^{p-2} u^{-}-b|u|^{p-2} u+s \frac{\phi_{1}}{\lambda_{1}-b}\right)=T u,
$$

where $M=\left(-L_{p}-b g_{p}\right)^{-1}$. The operator $M$ is compact on $L^{p}(\Omega)$, and the set $K=M(\bar{B})$, where $\bar{B}$ is the closed unit ball in $L^{p}(\Omega)$. Then $K$ is a compact set. Let us set $\gamma=\min \{b-$ $\left.\lambda_{2}, \lambda_{3}-b\right\}$. We can observe that $\|M(u)\|_{L^{p}(\Omega)} \leq \frac{1}{\gamma}\left\|g_{p}^{-1}(u)\right\|_{L^{p}(\Omega)}$. Let $\alpha$ be the modulus continuity given in Lemma 4.3 corresponding to $K$ and $\xi=M\left(s \phi_{1}^{p-1}\right)=\left(\frac{s \phi_{1}}{\lambda_{1}-b}\right)^{\frac{1}{p-1}} \phi_{1}$, and choose $\epsilon>0$ so that

$$
\left.\alpha\left((\epsilon(b-a))^{\frac{1}{p-1}}+\gamma\right)\right) \leq \frac{\gamma}{4(b-a)^{\frac{1}{p-1}}\left((b-a)^{\frac{1}{p-1}}+\gamma\right)} .
$$

We have

$$
\left\|b|u|^{p-2} u^{+}-a|u|^{p-2} u^{-}-b|u|^{p-2} u\right\|_{L^{p}(\Omega)} \leq(b-a)\left\||u|^{p-2} u^{-}\right\|_{L^{p}(\Omega)} .
$$

For $u \in\left(\frac{s}{\lambda_{1}-b}\right)^{\frac{1}{p-1}} \phi_{1}+(|s| \epsilon v)^{\frac{1}{p-1}}$ with $v \in \bar{B}$

$$
\left\|u^{-}\right\|_{L^{p}(\Omega)}=\left\|\left(\left(\frac{s}{\lambda_{1}-b}\right)^{\frac{1}{p-1}} \phi_{1}+(|s| \epsilon v)^{\frac{1}{p-1}}\right)^{-}\right\|_{L^{p}(\Omega)} \leq\left\|\left((|s| \epsilon \nu)^{\frac{1}{p^{-1}}}\right)^{-}\right\|_{L^{p}(\Omega)} \leq|s| \epsilon
$$

since $\left(\frac{s}{\lambda_{1}-b}\right)^{\frac{1}{p-1}} \phi_{1}>0$. Then $T(u)=M\left(b|u|^{p-2} u^{+}-a|u|^{p-2} u^{-}-b|u|^{p-2} u+s \frac{\phi_{1}}{\lambda_{1}-b}\right)$ can be rewritten as

$$
T(u)=\left(\frac{s}{\lambda_{1}-b}\right)^{\frac{1}{p-1}} \phi_{1}+(|s| \epsilon)^{\frac{1}{p-1}}\left((b-a)^{\frac{1}{p-1}}+\gamma\right) v, \quad v \in K .
$$


If $u$ is a solution of (4.4), then $u=T u$ and, by Lemma 4.3,

$$
\begin{aligned}
\left\|u^{-}\right\|_{L^{p}(\Omega)} & \left.=\|\left(\frac{s}{\lambda_{1}-b}\right)^{\frac{1}{p-1}} \phi_{1}+(|s| \epsilon)^{\frac{1}{p-1}}\left((b-a)^{\frac{1}{p-1}}+\gamma\right) \nu\right)^{-} \|_{L^{p}(\Omega)} \\
& \leq(|s| \epsilon)^{\frac{1}{p-1}}\left((b-a)^{\frac{1}{p-1}}+\gamma\right) \alpha\left(\epsilon^{\frac{1}{p-1}}\left((b-a)^{\frac{1}{p-1}}+\gamma\right)\right) \\
& <\frac{\gamma(|s| \epsilon)^{\frac{1}{p-1}}}{4(b-a)^{\frac{1}{p-1}}} .
\end{aligned}
$$

It follows that

$$
\begin{aligned}
\left\|M\left(b|u|^{p-2} u^{+}-a|u|^{p-2} u^{-}-b|u|^{p-2} u\right)\right\|_{L^{p}(\Omega)} & \leq(b-a)^{\frac{1}{p-1}}\left\|u^{-}\right\|_{L^{p}(\Omega)} \\
& \leq \frac{1}{4}(|s| \epsilon)^{\frac{1}{p-1}} \leq \frac{1}{4}|s| \epsilon .
\end{aligned}
$$

Thus we have shown that any solution $u \in\left(\frac{s}{\lambda_{1}-b}\right)^{\frac{1}{p-1}} \phi_{1}+|s| \epsilon \bar{B}$ of (4.4) belongs to $\left(\frac{s}{\lambda_{1}-b}\right)^{\frac{1}{p-1}} \phi_{1}+\frac{1}{4}|s| \epsilon \bar{B}$. This estimate holds if we replace $b|u|^{p-2} u^{+}-a|u|^{p-2} u^{-}-b|u|^{p-2} u$ by $\lambda\left(b|u|^{p-2} u^{+}-a|u|^{p-2} u^{-}-b|u|^{p-2} u\right)$ with $0 \leq \lambda \leq 1$. Thus the equation

$$
u=\left(-L_{p}\right)^{-1}\left(s \phi_{1}^{p-1}+b|u|^{p-2} u+\lambda\left(b|u|^{p-2} u^{+}-a|u|^{p-2} u^{-}-b|u|^{p-2} u\right)\right)
$$

has no solution on the boundary of the ball $B_{\epsilon|s|}\left(\left(\frac{s}{\lambda_{1}-b}\right)^{\frac{1}{p-1}} \phi_{1}\right)$ for $0 \leq \lambda \leq 1$. By the homotopy invariance degree,

$$
\begin{gathered}
d_{\mathrm{LS}}\left(u-\left(-L_{p}\right)^{-1}\left(s \phi_{1}^{p-1}+b|u|^{p-2} u+\lambda\left(b|u|^{p-2} u^{+}-a|u|^{p-2} u^{-}-b|u|^{p-2} u\right),\right.\right. \\
\left.B_{\epsilon|s|}\left(\left(\frac{s}{\lambda_{1}-b}\right)^{\frac{1}{p-1}} \phi_{1}\right), 0\right)
\end{gathered}
$$

is defined for $0 \leq \lambda \leq 1$ and is independent of $\lambda$. For $\lambda=0$,

$$
d_{\mathrm{LS}}\left(u-(-L)^{-1}\left(s \phi_{1}^{p-1}+b|u|^{p-2} u, B_{\epsilon|s|}\left(\left(\frac{s}{\lambda_{1}-b}\right)^{\frac{1}{p-1}} \phi_{1}\right), 0\right)=(-1) \times(-1)=+1,\right.
$$

since $u=\left(\frac{s}{\lambda_{1}-b}\right)^{\frac{1}{p-1}} \phi_{1}$ is the unique solution of the equation, and, since there are 2 eigenvalues $\lambda_{1}, \lambda_{2}$ of $-L_{p}$ to the left of $b$, the operator $I-b\left(-L_{p}\right)^{-1}$ has two negative eigenvalues, while all the rest are positive. When $\lambda=1$, we have

$$
\begin{aligned}
& d_{\mathrm{LS}}\left(\left(u-\left(-L_{p}\right)^{-1}\left(s \phi_{1}^{p-1}+b|u|^{p-2} u^{+}+1\left(b|u|^{p-2} u^{+}-a|u|^{p-2} u^{-}-b|u|^{p-2} u\right),\right.\right.\right. \\
& \left.\quad B_{\epsilon|s|}\left(\left(\frac{s}{\lambda_{1}-b}\right)^{\frac{1}{p-1}} \phi_{1}\right), 0\right) \\
& =d_{\mathrm{LS}}\left(s \phi_{1}^{p-1}+b|u|^{p-2} u^{+}-a|u|^{p-2} u^{-}, B_{\epsilon|s|}\left(\left(\frac{s}{\lambda_{1}-b}\right)^{\frac{1}{p-1}} \phi_{1}\right), 0\right) .
\end{aligned}
$$


Thus by the homotopy invariance of degree, we have

$$
\begin{aligned}
& d_{\mathrm{LS}}\left(s \phi_{1}^{p-1}+b|u|^{p-2} u^{+}-a|u|^{p-2} u^{-}, B_{\epsilon|s|}\left(\left(\frac{s}{\lambda_{1}-b}\right)^{\frac{1}{p-1}} \phi_{1}\right), 0\right) \\
& \quad=d_{\mathrm{LS}}\left(u-(-L)^{-1}\left(s \phi_{1}^{p-1}+b|u|^{p-2} u, B_{\epsilon|s|}\left(\frac{s}{\lambda_{1}-b}\right)^{\frac{1}{p-1}} \phi_{1}\right), 0\right)=1 .
\end{aligned}
$$

Thus the lemma is proved.

Lemma 4.5 Assume that $1<p<\infty,-\infty<a<\lambda_{1}, \lambda_{2}<b<\lambda_{3}$ and $s_{1}<0$. Then there exist a constant $\epsilon>0$ depending on $a, b$, such that for $s_{1} \leq s<0$, the Leray-Schauder degree

$$
d_{\mathrm{LS}}\left(u-(-L)^{-1}\left(b|u|^{p-2} u^{+}-a|u|^{p-2} u^{-}+s \phi_{1}^{p-1}\right), B_{|s| \epsilon}\left(u_{1}\right), 0\right)=1,
$$

where $u_{1}=-\left(\frac{s}{a-\lambda_{1}}\right)^{\frac{1}{p-1}} \phi_{1}<0$ is a negative solution of $(1.1)$.

Proof We can prove this lemma by an almost identical argument as that of Lemma 4.4.

Proof of Theorem 1.3 The proofs of Theorem 1.3(i)-(ii) are the same as those of Theorem 1.2(i)-(ii).

(iii) By Lemmas 4.4 and 4.5 , there is a solution $\left(\frac{s}{\lambda_{1}-b}\right)^{\frac{1}{p-1}} \phi_{1}>0$ in $B_{|s| \epsilon}\left(\left(\frac{s}{\lambda_{1}-b}\right)^{\frac{1}{p-1}} \phi_{1}\right)$ and a solution $-\left(\frac{s}{a-\lambda_{1}}\right)^{\frac{1}{p-1}} \phi_{1}<0$ in $B_{|s| \epsilon}\left(-\left(\frac{s}{a-\lambda_{1}}\right)^{\frac{1}{p-1}} \phi_{1}\right)$. We may assume that $\epsilon<\left(\frac{1}{b-\lambda_{1}}\right)^{\frac{1}{p-1}}$ and $\epsilon<\left(\frac{1}{\lambda_{1}-a}\right)^{\frac{1}{p-1}}$. Then these two balls are disjoint. This gives two solutions for any $n$. There is a large ball $B_{R}$ centered at the origin and containing $B_{|s| \epsilon}\left(\left(\frac{s}{\lambda_{1}-b}\right)^{\frac{1}{p-1}} \phi_{1}\right)$ and $B_{|s| \epsilon}\left(-\left(\frac{s}{a-\lambda_{1}}\right)^{\frac{1}{p-1}} \phi_{1}\right)$. Since

$$
\begin{aligned}
& d_{\mathrm{LS}}\left(u-\left(-L_{p}\right)^{-1}\left(b|u|^{p-2} u^{+}-a|u|^{p-2} u^{-}+s \phi_{1}^{p-1}\right), B_{R}(0), 0\right)=0 \\
& d_{\mathrm{LS}}\left(u-\left(-L_{p}\right)^{-1}\left(b|u|^{p-2} u^{+}-a|u|^{p-2} u^{-}+s \phi_{1}^{p-1}\right), B_{R}\left(\left(\frac{s}{\lambda_{1}-b}\right)^{\frac{1}{p-1}} \phi_{1}\right), 0\right) \\
& \quad=d_{\mathrm{LS}}\left(u-\left(-L_{p}\right)^{-1}\left(b|u|^{p-2} u^{+}-a|u|^{p-2} u^{-}+s \phi_{1}^{p-1}\right), B_{R}\left(-\left(\frac{s}{a-\lambda_{1}}\right)^{\frac{1}{p-1}} \phi_{1}\right), 0\right)=1
\end{aligned}
$$

we have

$$
\begin{aligned}
& d_{\mathrm{LS}}\left(u-(-L)^{-1}\left(b|u|^{p-2} u^{+}-a|u|^{p-2} u^{-}+s \phi_{1}^{p-1}\right),\right. \\
& \quad B_{R}(0) \backslash\left(B_{|s| \epsilon}\left(\left(\frac{s}{\lambda_{1}-b}\right)^{\frac{1}{p-1}} \phi_{1}\right) \cup B_{|s| \epsilon}\left(-\left(\frac{s}{\lambda_{1}-a}\right)^{\frac{1}{p-1}} \phi_{1}\right), 0\right) \\
& =-2 .
\end{aligned}
$$

Thus there exists a third solution in $B_{R}(0) \backslash\left(B_{|s| \epsilon}\left(\left(\frac{s}{\lambda_{1}-b}\right)^{\frac{1}{p-1}} \phi_{1}\right) \cup B_{|s| \epsilon}\left(-\left(\frac{s}{a-\lambda_{1}}\right)^{\frac{1}{p-1}} \phi_{1}\right)\right.$. Thus we have proved Theorem 1.3(iii). 
Acknowledgements

Not applicable

\section{Funding}

Tacksun Jung was supported by Basic Science Research Program through the National Research Foundation of Korea (NRF) funded by the Ministry of Science, ICT and Future Planning (NRF-2017R1A2B4005883). Q-Heung Choi was supported by Basic Science Research Program through the National Research Foundation of Korea (NRF) funded by the Ministry of Education, Science and Technology (2017R1D1A1B03030024).

\section{Abbreviations}

Not applicable

\section{Availability of data and materials}

Not applicable

\section{Competing interests}

The authors declare that there is no conflict of interests regarding the publication of this paper.

Authors' contributions

TJ introduced the main ideas of multiplicity study for this problem. Q-HC participated in applying the method for solving this problem and drafted the manuscript. All authors contributed equally to reading and approving the final manuscript.

\section{Author details}

'Department of Mathematics, Kunsan National University, Kunsan, Korea. ${ }^{2}$ Department of Mathematics Education, Inha University, Incheon, Korea.

\section{Publisher's Note}

Springer Nature remains neutral with regard to jurisdictional claims in published maps and institutional affiliations.

\section{Received: 25 August 2018 Accepted: 7 March 2019 Published online: 18 March 2019}

\section{References}

1. Choi, Q.H., Jung, T:: An application of a variational reduction method to a nonlinear wave equation. J. Differ. Equ. 117, 390-410 (1995)

2. Choi, Q.H., Jung, T:: A nonlinear suspension bridge equation with nonconstant load. Nonlinear Anal. TMA 35, 649-668 (1999)

3. Choi, Q.H., Jung, T.: Multiplicity results for the nonlinear suspension bridge equation. Dyn. Contin. Discrete Impuls. Syst., Ser. A Math. Anal. 9, 29-38 (2002)

4. Choi, Q.H., Jung, T., McKenna, P.J.: The study of a nonlinear suspension bridge equation by a variational reduction method. Appl. Anal. 50, 73-92 (1993)

5. del Pino, M., Elgueta, M., Manasevich, R.: A homotopic deformation along $p$ of a Leray-Schauder degree result and existence for $\left(\left|u^{\prime}\right|^{p-2} u^{\prime}\right)^{\prime}+f(x, u)=0, u(0)=u(T)=0, p>1$. J. Differ. Equ. 80, 1-13 (1998)

6. Ghergu, M., Rádulescu, V.: Existence and nonexistence of entire solutions to the logistic differential equations. Abstr. Appl. Anal. 2003(17), 995-1003 (2003)

7. Ghergu, M., Rádulescu, V:: Singular Elliptic Problems, Bifurcation and Asymptotic Analysis. Oxford Lecture Series in Mathematics and Its Applications. Oxford University Press, London (2008)

8. Kim, Y.-H., Wang, L., Zhang, C.: Global bifurcation for a class of degenerate elliptic equations with variable exponents. J. Math. Anal. Appl. 371, 624-637 (2010)

9. Lê, A.: Eigenvalue problems for the $p$-Laplacian. Nonlinear Anal. 64, 1057-1099 (2006)

10. Manásevich, R., Mawhin, J.: Periodic solutions for nonlinear systems with $p$-Laplacian-like operators. J. Differ. Equ. 145, 367-393 (1998)

11. Manásevich, R., Mawhin, J.: Boundary value problems for nonlinear perturbations of vector $p$-Laplacian-like operators J. Korean Math. Soc. 37, 665-685 (2000)

12. McKenna, P.J., Walter, W.: Nonlinear oscillations in a suspension bridge. Arch. Ration. Mech. Anal. 98, 167-177 (1987)

13. O'Regan, D.: Some general existence principles and results for $\left(\phi\left(y^{\prime}\right)\right)^{\prime}=q f\left(t, y, y^{\prime}\right), 0<t<1$. SIAM J. Math. Anal. 24, 648-668 (1993) 